\title{
Being direct
}

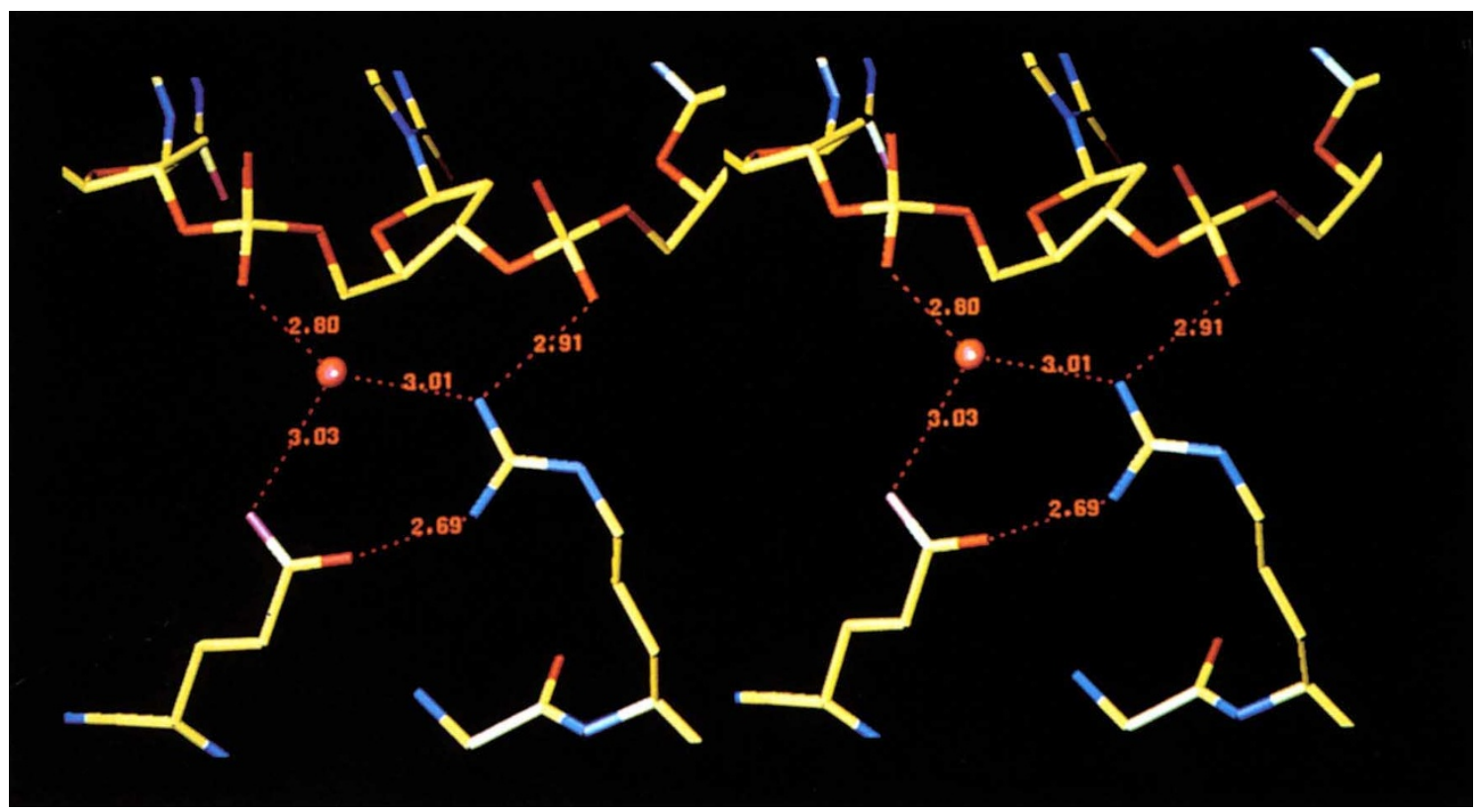

Recent years have seen an explosion in the number of X-ray structures for protein-DNA complexes. Many of these structures feature water molecules buried at the intermolecular interface. Otherwise unaccounted for electron density at an interface can be assigned with reasonable confidence as a water oxygen atom if the coordination sphere of hydrogen bond donors and acceptors exhibits good geometry. The temperature factors for such waters can be similar to those of the functional groups that contribute the donors and acceptors. Thus, hydrogen-bonding networks between protein and DNA often include intervening water, a result that was not anticipated from our previous understanding of the molecular basis for macromolecular recognition, which predicted direct contact between functional groups.

A second surprise, which arose from X-ray structures of defined-sequence DNA molecules, is that recognition can be indirect for a reason that is fundamentally unrelated to the presence of water. Our previous view of recognition predicted that the sequence information content of the DNA was contained exclusively in the basepair functional groups accessible in the major or minor groove. The chemically degenerate phosphodiester backbone was thought incapable of transmitting sequence-dependent information to a binding protein. The first single-crystal DNA structures sometimes revealed extreme distortions of the backbone away from canonical helical parameters in a manner that clearly depended on nucleotide sequence. Thus, backbone contacts can be used by a binding protein to detect the DNA sequence indirectly. Such contacts, like those to the basepair edges, can be either water-mediated or direct. There is a potential for semantic confusion here: DNA sequence can be detected directly or indirectly, using either direct or indirect contacts, but there is no necessary correlation between the nature of the contacts and the nature of the detection mechanism. Many of the complexes examined so far present all four possible combinations. Illustrated here is an example from the refined 1.9 A structure of the DNA complex of TATA-binding protein (J.L. Kim \& S.K. Burley, Nature Structura/ Biology 1, 638-651). One arginine side chain makes a direct contact to one phosphate oxygen of the backbone and a watermediated contact to the next phosphate; the position of the arginine side chain is reinforced by one direct and one water-mediated contact to a neighboring glutamate side chain. Regardless of whether they are direct of water-mediated, or backbone contacts cannot be inferred to have a role in indirect sequence recognition without assessing the local DNA conformation. The contacts depicted occur in the region of greatest distortion of the DNA structure; if these distortions are sequence-dependent, then these phosphate contacts convey sequence information to the protein.

Finally, it is not possible to tell by inspection whether an observed contact is specificity-determining. Specificity is essentially a thermodynamic issue and thus not amenable to structural analysis alone. A useful operational measure of specificity is the relative affinities of two targets that differ by a particular functional group change. Affinity must be measured in a binding experiment, preferably at equilibrium, although for very strong interactions, this presents a technical challenge. The affinity between binding molecules is the net result of favourable and unfavourable energetic contributions to the free and bound states, the magnitudes of which can be very large relative to the differences between them. Thus, offsetting changes are not unexpected when a functional group is altered, and equivalent affinities for two targets doesn't necessarily imply that the group makes no contribution to specific recognition. Structural analysis of the targets and the protein in both free and bound states is therefore crucial, although cryptic energetic contributions from conformational flexibility and adaptation can also be important determinants of specificity.

Jannette L. Carey \& Teresa A. Lanoie Department of Chemistry, Princeton, New Jersey 08544-1009, USA 